\title{
Verification of Himawari-8 Observation Data using Cloud Optical Thickness (COT) and Cloud Image Energy
}

\author{
Umar Ali Ahmad $^{1}$, Alex Lukmanto Suherman ${ }^{5}$ \\ Agus Virgono $^{8}$, Burhanuddin Dirgantoro ${ }^{9}$ \\ Reza Rendian Septiawan ${ }^{10}$ \\ Department of Computer Engineering, School of Electrical \\ Engineering, Telkom University, Bandung, Indonesia \\ Wendi Harjupa ${ }^{2}$ \\ Center of Atmospheric Science and Technology \\ LAPAN, Bandung, Indonesia \\ Dody Qory Utama ${ }^{3}$ \\ School of Informatics. Telkom University \\ Bandung, Indonesia
}

\author{
Risyanto $^{4}$, Prayitno Abadi ${ }^{7}$ \\ Space Science Center. LAPAN \\ Bandung, Indonesia
}

\author{
Wahyu Pamungkas ${ }^{6}$ \\ Fakultas Teknik Telekomunikasi dan Elektro \\ Institut Teknologi Telkom \\ Purwokerto, Indonesia
}

Mas'ud Adhi Saputra ${ }^{11}$

Center for Material and Technical Products

Ministry of Industry, Jakarta

Indonesia

\begin{abstract}
Himawari-8 satellite cloud observation data covers all areas of Indonesia. The cloud observation data can be used for observations of current weather conditions and short-term predictions. This paper reports the verification method of Himawari-8 Observation Data using Cloud Optical Thickness (COT) and compared to Cloud Image Energy. The verification test was carried out to determine the accuracy of Himawari-8's observations. COT data were verified using energy data from the observation image of the time-lapse camera. First, the time-lapse camera captures and classifies the cloud image. Subsequently, the energy of each image frame was calculated and re-grouped the result based on the energy to determine the type of the cloud. The results show that there is a positive correlation between COT and low energy values with cumulonimbus cloud detection, on the contrary for Cirrus-cloud type. However, the data requires a more accurate observation method to obtain data from cloud images on the Himawari-8 satellite, specifically for regions with a small spatial size of $\mathbf{4} \mathbf{~ k m}$ and thin clouds in the lower layer.
\end{abstract}

Keywords-Himawari-8; COT; image classification; cloud energy

\section{INTRODUCTION}

Rain formation is not an easy phenomenon to understand, although it can be simplified. Rain can occur from the process of evaporation, cloud formation (condensation), and the dropping of water from the atmosphere (precipitation). However, in fact, the process of rain formation becomes complicated as well as the process of forming clouds. A combination of atmospheric parameters, such as temperature, pressure, and humidity at a certain value, can produce clouds.

The next question is whether the formed clouds will certainly produce rain or not. Weather prediction, such as the prediction of extreme rain in Indonesia, is a very challenging matter. Indonesia's geographic location, which is located between two continents (Asia and Australia) and two oceans (Indian and Pacific), causes Indonesia to be traversed by many global circulations. For example, the Madden-Julian Oscillation (MJO) and Monsoon circulations. In addition, Indonesia's hilly topography causes local circulation, such as convection to be very active, which also has an impact on the weather.

Recent progress in the satellite cloud technologies, such as Himawari-8, enable us to predict the current weather and short-term conditions. Himawari-8 is a geostationary weather satellite that launched by the Japan Meteorology Agency (JMA) in 2014. Himawari-8 is equipped with the Advanced Himawari Imager (AHI) instrument with a significant increase in the number of radiometric spectral channels and spatial resolution, compared to its previous generations [1]. The number of AHI observation channels consists of three Visible channels, three Near Infra-Red (NIR) channels, and 10 InfraRed (IR) channels, with a spatial resolution of 0.5 and $1 \mathrm{~km}$ for Visible and NIR, and $2 \mathrm{~km}$ for IR in Japan [1].

Research using AHI has also been applied by Letu et al. [2] by using the Look-Up Table (LUT) method to calculate high temporal (10 minutes) and high spatial (5 km) of surface solar radiation from AHI cloud properties. AHI properties, especially those related to ice cloud products, were also used by Letu et al. [2] to monitor the growth process, including monitoring variations of cloud properties and deposition in Deep Convective (DC) clouds. Research conducted by Lee et al. [3] also uses AHI, which is sensitive to clouds and gas absorption, to improve the algorithm for calculating Outgoing Longwave Radiation (OLR) at the peak of the atmosphere. 
In Indonesia, the Himawari-8 satellite observations cover the entire area from Western to Eastern parts of Indonesia with time and space resolutions of 10 minutes and $4 \mathrm{~km}$. Several researches using Himawari-8 satellite [4][5] are used to detect the condition of volcanoes in Indonesia. Another application of the Himawari-8 satellite in Indonesia was carried out by Osawa et al. [6], researching Long Internal Solitary Waves (ISWs), which seem to be generated from the Sape Strait, which then pro-gates through the Java Sea, the Sumba Strait, and the Savu Sea. In a country directly adjacent to Indonesia, namely Papua, Himawari-8 is also used to investigate the topography of the country's territory [7]. These reported researches indicate the advantages of using the Himawari-8 satellite to monitor cloud activity near real-time.

The data verification method is crucial to maximize the use of the Himawari-8 Satellite data. One example of the use of Himawari-8 data is for rain estimation. Cloud Optical Thickness (COT) can provide qualitative cloud thickness information. Concerning the decrease in solar radiation, COT has an exponential value, where COT and solar radiation data were taken from Nanyang Technological University (NTU) for four months [8].

Research that examines the effect of COT, the albedo of ground surface, and the above-cloud absorbing dust layer structure result in the phenomenon that higher reflectance is polarized along with the increase in COT and will be saturated when COT = 10 [9]. Meanwhile, the Cloud Optical ThicknessRetrieval Over Snow (CROS) algorithm has been used in snowy areas [10] to tackle this issue, but the issue of estimation accuracy is crucial. Information on cloud thickness is important in estimating rainfall [11]. Sakai et al. [12] used cloud observations from the Himawari-8 satellite to produce COT. Harjupa et al. [13] used cloud data from the Himawari-8 to predict the extreme rain within the next hour (nowcasting). However, Himawari-8 satellite cannot observe multi-layered clouds and thus the accuracy of COT and predictive products becomes our concern. The aim of this paper is to tackle this limitation. For this reason, COT verification is carried out with data from camera observations from the earth's surface. In this paper, we are using a lapse-time camera which can take image of clouds with high time and spatial resolution. Observations from the earth's surface are needed to observe clouds in the lower layers.

This paper consists of the following sections. In Section II, we define our method to observe the cloud properties and illustrate the process of classification and detection using the time-lapse camera. In Section III, we present the cloud grouping from a time-lapse camera based on the energy level, compared with the images from the satellite. In Section IV, we discussed the results presented in the previous chapter in a structured manner. In Section V, the results are concluded and further research plans are presented.

\section{Materials AND Methods}

Fig. 1 shows cloud detection method using the Himawari-8 satellite, which is carried out from space and cloud detection using a camera from the earth's surface. The results suggest that the observations from the satellites can only detect clouds in the upper layers, not the lower layers. Camera observations made from the earth's surface will complement the cloud observation data from the Himawari-8 satellite.

The results from cloud observation using a Himawari-8 satellite will be derived in the form of a COT product. The cloud observation image will be analyzed and the energy value of the cloud will be calculated. The two observations was performed and compared to determine the accuracy of the Himawari-8 observations. Fig. 2 shows data verification diagram for the Himawari-8 and the camera.

\section{A. Cloud Top Layer Thickness}

The COT value in this study was obtained from the difference in the value of brightness temperature (BT) of band13 and brightness temperature difference (BTD) of band15 observed by Himawari-8. The height of the cloud can be measured qualitatively using band13, where the lower the brightness of the temperature band13 indicates the high of the cloud peak due to the reduced surface radiation value. Nishi et al [14] found that at BT value of $220 \mathrm{~K}$, the cloud height is about $14.4 \mathrm{~km}$. By comparing the BT value between band13 and band15, the COT value can be determined. The COT value will be bigger at the higher cloud positions [12].

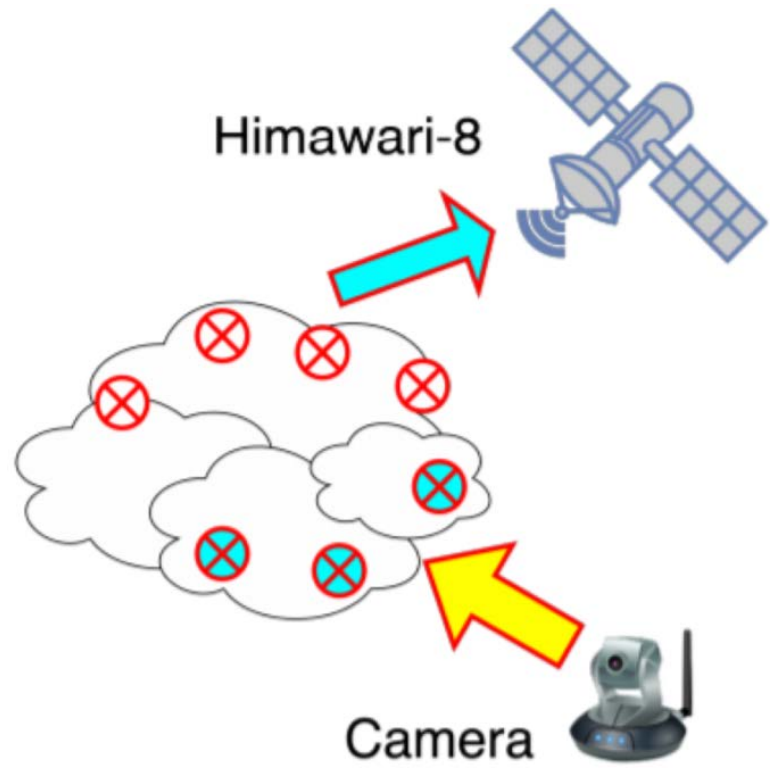

Fig. 1. Cloud Observation using the Himawari-8 Satellite and a Time-Lapse Camera.

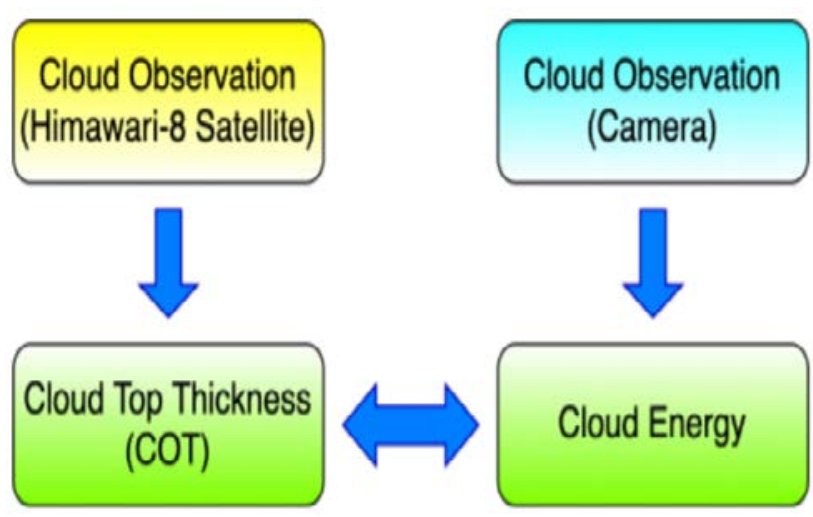

Fig. 2. Comparison of COT and Cloud Energy. 
The COT value, which is the difference in the BT value of the two bands (BTD), can be used to classify the types of rain clouds [15]. The cloud types of the two BT are shallow and non-shallow clouds. With these results, the COT value will be beneficial for estimating the rain generated by clouds. In this study, the location and time of cloud detection were selected manually, namely by matching the same time and place with the cloud observed from the time-lapse camera.

\section{B. Classification and Cloud Detection using Time-Lapse Camera}

Fig. 3 shows how we classify the clouds from time-lapse camera. First step is converting the time-lapse video images into several images, which will be analyzed and classified. Following this, the energy of each frame of the image were calculated. Subsequently, each cloud was classified based on the energy value obtained for each frame.

The framing process in Fig. 4 aims to take a sample of time unity from the existing time-lapse video. This process was carried out since the movement of the cloud is slow enough for the unity of time. It can be concluded that the near union time can be ascertained that the cloud will have adjacent energy. In this study, the framing process was performed by taking one digital image frame every ten frames, with 30 frames per second.

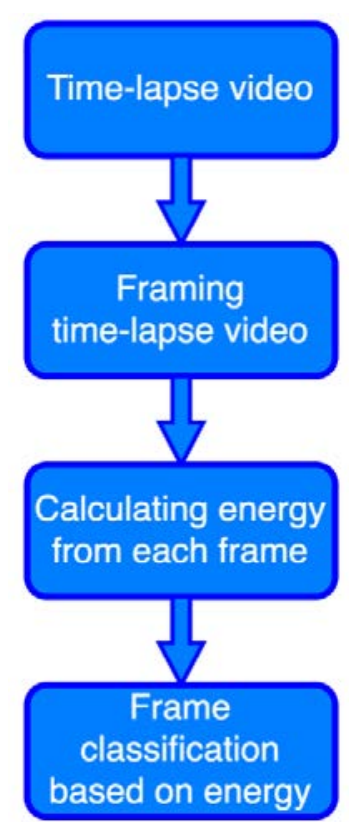

Fig. 3. The Cloud Classification Method using a Time-Lapse Camera.

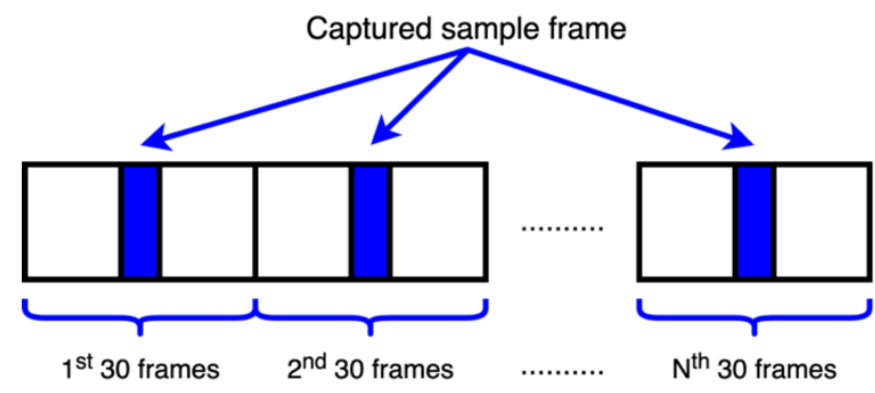

Fig. 4. Video Framing Method Lapse-Time.
The average brightness of each frame obtained from the framing process was used to calculate the energy levels. This process can be seen in Fig. 5.

The frame (a digital image) will indicates the brightness value for each pixel, then add up all the brightness values and divided by the calculated pixel count, as shown in the following mathematical notation:

Frame energy $=\frac{\sum_{x=0}^{n} \operatorname{pixel}(x)}{n}$

After getting the energy level for each frame, the frames will be grouped according to seven energy values, namely group 1 (energy of 0-20), group 2 (energy of 20-40), group 3 (energy of 40-60), group 3 (energy of 60-80), group 4 (energy of 80-100), group 5 (energy of 100-120), group 6 (energy of 120-140), and group 7 (energy above 140). The energy level will determine the type of cloud. Fig. 6 shows the grouping of frames based on energy levels.

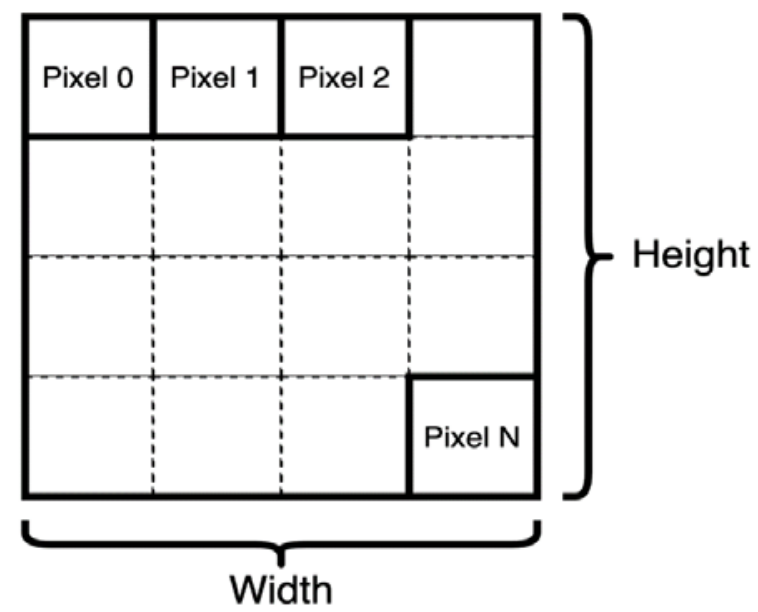

Fig. 5. Frame Structure when Measuring its Energy Level.

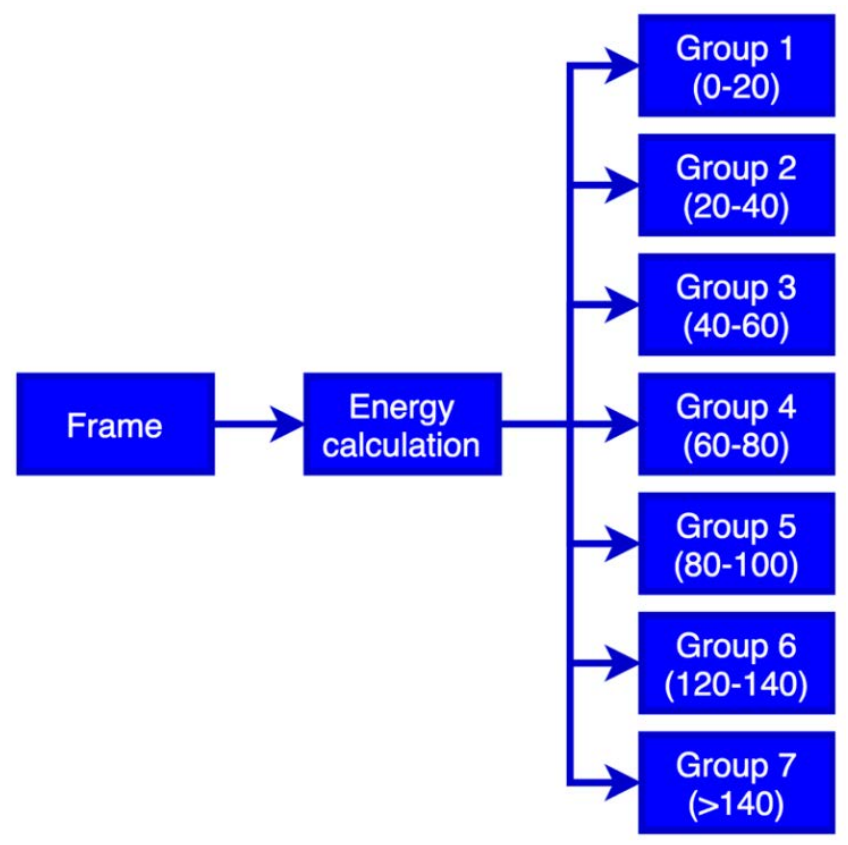

Fig. 6. Grouping of the Frame’s Energy. 


\section{RESUltS}

Table I shows the image resulted from the time-lapse camera, showing various sample images belong to the respective cloud energy groups. Comparison of COT values and cloud energy is shown.

TABLE I. The Results of ClOUd GROUPING FROM A TIME-LAPSE CAMERA BASED ON THE ENERGY LEVEL

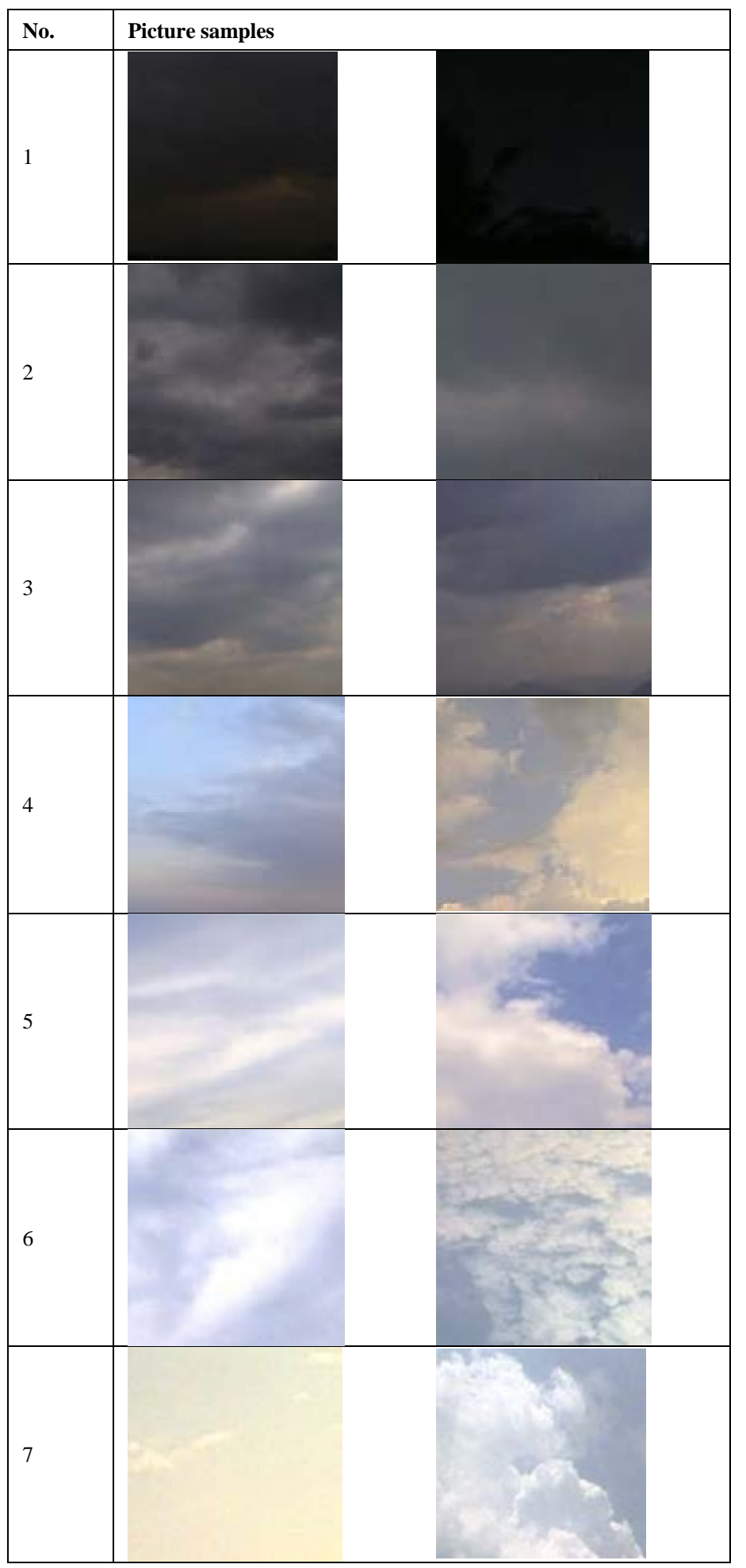

The comparison of the COT value and cloud energy is shown in Table II. The COT value is taken in the yellow circle area, which is the same area as the cloud observed by the time-lapse camera.

TABLE II. THE RESULTS OF CLOUD GROUPING FROM A TIME-LAPSE CAMERA COMPARED WITH THE PICTURE FROM THE SATELLITE

\begin{tabular}{|c|c|c|c|}
\hline COT & $\begin{array}{l}\text { Picture samples from } \\
\text { satellite }\end{array}$ & $\begin{array}{l}\text { Energy } \\
\text { level }\end{array}$ & $\begin{array}{l}\text { Picture samples from the } \\
\text { camera }\end{array}$ \\
\hline 1.16 & & 138 & \\
\hline 3.35 & & 110 & \\
\hline 0.55 & & 126 & \\
\hline 9.31 & & 161 & \\
\hline nan & & 153 & \\
\hline nan & & 213 & \\
\hline 1.99 & & 51 & \\
\hline
\end{tabular}

\section{DISCUSSIONS}

\section{A. Energy-based Grouping of Clouds from Time-Lapse Camera}

In group 1, with an energy value of $0-20$, the cloud conditions are very dark. It was found that the value of cloud energy becomes very small at sunset or night, as shown in Table I. From the grouping clouds data based on this energy, it is found that clouds with different types can produce the same 
energy as seen in the cloud, with energy group 5, 6 and 7. Types of clouds in the energy group 5 are Stratus and Cirrus clouds. The same type of clouds is found in group 6 and 7. In addition, the same type of clouds also found from the Himawari-8 observations, where a small value of brightness temperature (BT) could be produced by Cirrus, Stratus or Cumulus-type clouds.

\section{B. Cloud Energy from the Camera and COT of Himawari-8}

COT values and cloud energy in group 1 in Table II is 1.16 and 138, respectively. Since the found energy level is 138, it is a Stratus-type cloud and not too thick. Clouds with lower energy values are seen in the comparison of the COT value and cloud energy level of group 2 in Table II. The initial thickness makes the detected energy lower, namely, 110, and this value is comparable to the COT value, which is higher than the COT value in comparison with number 1 . Low energy and COT values indicate a thick cloud that may produce a heavy rain. In comparison, number 3 in the same figure, it can be seen that the COT value is very low, namely 0.55 , with a high energy value of 126 . The type of cloud in the ratio number 3 are tall and thin clouds, like Cirrus-cloud type. In comparison, number 4 shows a difference where the high COT value is 9.31, but the energy value is also high, namely 161. The difference in COT values results an error during the prediction of heavy rain. With the help of time-lapse camera, the results of cloud observations from the Himawari-8 satellite can be validated. In comparison between number 5 and 6 , no COT value is detected, while the camera has energy values, namely 153 and 213. From the image of the cloud that is extracted, the energy shows that it is spatially and the observed cloud area is not very large. This is a weakness of Himawari-8's observations, as it cannot observe clouds with a spatial area smaller than $4 \mathrm{~km}$. This result shows the important aspect of using a time-lapse camera, as it can detect clouds with a smaller spatial resolution. In comparison, number 7 shows that the COT value is not too high with a low energy value, suggesting the cloud type is a thin cloud or Cirrus cloud-type.

\section{CONCLUSIONS}

In this initial verification of Himawari-8 observation data and cloud energy from camera observations, several conclusions were obtained: (a) high COT values and low energy values indicate cumulonimbus cloud detection, on the contrary for cirrus cloud-type, the energy value will be high with low COT, (b) the Himawari-8 satellite cannot capture cloud images with a small spatial size of $4 \mathrm{~km}$ and thin clouds in the lower layer, (c) it is necessary to establish a relationship between the COT value and the energy value of each cloud type to tackle the limitation of Himawari-8 observation data and to increase the capability or accuracy of derivative product such rain prediction using Himawari-8's observation data.

\section{FUTURE WORKS}

Further experimental studies are required for validation of the proposed method. The increasing dataset will have the advantage to increase the result validity throughout a longer period of observation. Furthermore, the relation information must be expanded to other research fields, such as weather prediction.

\section{ACKNOWLEDGMENTS}

This research was supported by Telkom University, the Center of Atmospheric Science and Technology of LAPAN, and the Space Science Center of LAPAN, Indonesia.

\section{REFERENCES}

[1] K. Bessho, et al., "An introduction to Himawari-8/9 — Japan's newgeneration geostationary meteorological satellites,” J. Meteorol. Soc. Japan. Ser. II, 94(2), 2016, pp. 151-183. DOI: 10.2151/jmsj.2016-009.

[2] H. Letu, et al., "Ice Cloud Properties From Himawari-8/AHI NextGeneration Geostationary Satellite: Capability of the AHI to Monitor the DC Cloud Generation Process,” IEEE Trans. Geosci. Remote Sens., 57(6), 2019, pp. 3229-3239. DOI: 10.1109/TGRS.2018.2882803.

[3] B. Y. Kim and K. T. Lee, "Using the Himawari-8 AHI multi-channel to improve the calculation accuracy of outgoing longwave radiation at the top of the atmosphere," Remote Sens., 11(5), 2019. DOI: 10.3390/rs11050589.

[4] F. Marchese, et al., "Monitoring the Agung (Indonesia) ash plume of November 2017 by means of infrared Himawari 8 data," Remote Sens., 10(6), 2018. DOI: 10.3390/rs10060919.

[5] T. Kaneko, et al., "Himawari-8 infrared observations of the June-August 2015 Mt Raung eruption, Indonesia,” Earth Planets Space. Springer Berlin Heidelberg, 70(1), 2018, pp. 1-9. DOI: 10.1186/s40623-0180858-9.

[6] I. W. G. A. Karang, Chonnaniyah, and T. Osawa, "Internal solitary wave observations in the Flores Sea using the Himawari-8 geostationary satellite,” Int. J. Remote Sens.. Taylor \& Francis, 41(15), 2020, pp. 5726-5742. DOI: 10.1080/01431161.2019.1693079.

[7] H. Iwabuchi et al., "Cloud property retrieval from multiband infrared measurements by Himawari-8,” J. Meteorol. Soc. Jpn., 96B(September 2017), 2018, pp. 27-42. DOI: 10.2151/jmsj.2018-001.

[8] F. Yuan et al., "Correlation between cloud optical thickness and solar radiation,” 2016 USNC-URSI Radio Science Meeting (Joint with AP-S Symposium), USNC-URSI 2016 - Proceedings, (1), 2016, pp. 105-106. DOI: 10.1109/USNC-URSI.2016.7588534.

[9] H. Shang, et al., "The effect of cloud optical thickness, ground surface albedo and above-cloud absorbing dust layer on the cloudbow structure," 2016 IEEE International Geoscience and Remote Sensing Symposium (IGARSS), Beijing, 2016, pp. 2174-2176, DOI: 10.1109/IGARSS.2016.7729561.

[10] C. Schlundt, et al., "Determination of cloud optical thickness over snow using satellite measurements in the oxygen A-band," IEEE Geosci. Remote. Sens. Lett., 10(5), 2013, pp. 1162-1166. DOI: 10.1109/LGRS.2012.2234720.

[11] S. Chakraborty and A. Maitra, "Interrelation between microphysical and optical properties of cloud and rainfall in the Indian region," Indian J. Radio and Space Phys., 42, 2013, pp. 105-112.

[12] Sakai, et al., "Development of a Rapid Retrieval Method for Cloud Optical Thickness and Cloud-Top Height using Himawari-8 Infrared Measurements,” Sci. Online Lett. Atmosphere, 15, 2019, pp. 57-61.

[13] W. Harjupa, et al., "Fundamental Investigation of Generation of Guerilla-heavy rainfall using Himawari-8 and XRAIN information in Kinki region,” J. Hydraul. Eng., JSCE, 74(4), 2017, pp. 283-288.

[14] A. Hamada and N. Nishi, "Development of a Cloud-Top Height Estimation Method by Geostationary Satellite Split-Window Measurements Trained with CloudSat Data." J. Appl. Meteor. Climatol., 49 (9), 2010, pp. 2035-2049.

[15] D. So and D. B. Shin, " Classification of precipitating clouds using satellite infrared observations and its implications for rainfall estimation," Advances in Remote Sensing of Rainfall and Snowfall, 2018. https://doi.org/10.1002/qj.3288. 